\title{
A GLUING LEMMA AND OVERCONVERGENT MODULAR FORMS
}

\author{
PAYMAN L KASSAEI
}

\begin{abstract}
We prove a gluing lemma for sections of line bundles on a rigid analytic variety. We apply the lemma, in conjunction with a result of Buzzard's $\mathrm{Bu}$, to give a proof of (a generalization of) Coleman's theorem which states that overconvergent modular forms of small slope are classical. The proof is "geometric" in nature, and is suitable for generalization to other Shimura varieties.
\end{abstract}

\section{INTRODUCTION}

Let $p$ be a prime number, $N \geq 5$ an integer prime to $p$, and $m \geq 1$ and $k$ integers. Let $K$ be a finite extension of $\mathbb{Q}_{p}$. Let $X_{1}\left(N p^{m}\right)_{K}$ denote the modular curve of level $\Gamma_{1}\left(N p^{m}\right)$ over $K$, and let $\underline{\omega}$ be the usual invertible sheaf on $X_{1}\left(N p^{m}\right)_{K}$ which on the non-cuspidal locus is the push-forward of the sheaf of invariant differentials of the universal elliptic curve. Let $X_{1}\left(N p^{m}\right)_{K}^{\text {an }}$ denote the rigid analytic version of $X_{1}\left(N p^{m}\right)_{K}$, and denote the analytification of $\underline{\omega}$ again by $\underline{\omega}$. An overconvergent $p$-adic modular form of level $\Gamma_{1}\left(N p^{m}\right)$ and weight $k$ defined over $K$ is a section of $\underline{\omega}^{k}$ on a rigid analytic subdomain of $X_{1}\left(N p^{m}\right)_{K}^{\text {an }}$ which strictly contains the component of the ordinary locus containing the cusp $\infty$. The subspace of classical modular forms consists of those sections of $\underline{\omega}^{k}$ which can be extended to $X_{1}\left(N p^{m}\right)_{K}^{\text {an }}$. By the rigid analytic GAGA, any such section is the analytification of an honest modular form (i.e., a section of $\underline{\omega}^{k}$ on $\left.X_{1}\left(N p^{m}\right)_{K}\right)$. In Co1, Co2, Coleman proves the following.

Theorem 1.1. (Coleman [Co1, Co2 $)$. Let $f$ be an overconvergent $\mathrm{U}_{p}$-eigenform of weight $k$ with eigenvalue $a_{p}$. If the $p$-adic valuation of $a_{p}$ is less than $k-1$, then $f$ is classical.

This so-called "control theorem" is crucial in many applications of the theory to modular forms. In many problems, one can reduce the "rigidity" of the situation by working in Banach spaces of overconvergent forms, and yet in the end get results about classical modular forms, by invoking the control theorem. For example, it is easier to construct $p$-adic families of overconvergent eigenforms, and then identify classical members of these families. One is interested in such control theorems for automorphic forms over other Shimura varieties. For instance, such a result is still missing for (non-ordinary) overconvergent Hilbert modular forms (See KL). Coleman's method of proving Theorem 1.1. which is based on a cohomological interpretation of the space of overconvergent forms (modulo the image of $\theta^{k-1}$ ), appears difficult to be carried out in more general situations (though it has the advantage that it gives some information about the case of critical slope.)

The purpose of this paper is to present a more intrinsic proof of (a generalization of) Theorem 1.1 which could serve as a model for extension to other Shimura varieties. In keeping with this goal, we have tried to use the specifics of the situation as little as possible. For instance, we use $q$-expansions only to show that modular forms are analytic at cusps.

In an upcoming article, we used this method to prove a control theorem for overconvergent modular forms over other Shimura curves (see Kas1, Kas2] for the general theory of overconvergent modular forms over these Shimura curves). We expect this method to 
apply without much trouble in some higher dimensional settings (for instance, over unitary Shimura varieties which arise in the work of Harris and Taylor $[\mathrm{HT}]$ ). The applicability of this method in the case of Hilbert modular varieties, however, seems to be contingent upon a better understanding (than at the moment) of the canonical subgroups of HilbertBlumenthal Abelian varieties. It also appears that our method can be applied in the context of the theory of overconvergent modular forms of half-integral weight developed by Nick Ramsey.

We were inspired by the work of Buzzard and Taylor [BT] to pursue the strategy of analytic continuation of modular forms. The subsequent paper of Buzzard [Bu] gave us the right starting point. He proves that an overconvergent $\mathrm{U}=\mathrm{U}_{p}$ eigenform $f$ with non-zero eigenvalue $a=a_{p}$ can be extended over the supersingular locus to a "big" admissible open subset of the modular curve. Under the assumption $v(a)<k-1$, we prove the classicality of $f$ by showing that $f$ can be extended further, over the complement of this admissible open. We prove the following generalization of Theorem 1.1

Theorem 1.2. Let $f$ be an overconvergent modular form of weight $k$ and level $\Gamma_{1}\left(N p^{m}\right)$ defined over $K$. Let $R(x) \in K[x]$ be a polynomial all roots of which in $\mathbb{C}_{p}$ have $p$-adic valuation less than $k-1$. If $R(\mathrm{U}) f$ is classical, then so is $f$.

Let us explain the steps involved in the proof of Theorem 1.2 in some detail. For the purpose of presentation, we will take $m=1, R(x)=x-a, v(a)<k-1$, and we assume $R(\mathrm{U}) f=0$, i.e. $\mathrm{U} f=a f$. Let $\mathcal{Z}^{\infty}(N p)$ (resp., $\mathcal{Z}^{0}(N p)$ ) denote the connected component of the ordinary part of $X_{1}(N p)_{K}^{\text {an }}$ which contains the cusp $\infty$ (resp., 0). By Buzzard's work $\mathrm{Bu}$. one can extend $f$ to $\mathcal{U}_{1}(N p)$ which is the rigid analytic part of $X_{1}(N p)_{K}^{\text {an }}$ whose non-cuspidal points consist of all $(E, i, P)$ where $E$ is an elliptic curve, $i$ is a level $\Gamma_{1}(N)$-structure, and $P$ is a point of order $p$ on $E$, and either $E$ has supersingular reduction, or $E$ has ordinary reduction and $P$ generates the canonical subgroup of $E$ (or equivalently $\left.(E, i, P) \in \mathcal{Z}^{\infty}(N p)\right)$.

To show that $f$ is classical, we have to show that $f$ can be further extended to the missing part $X_{1}(N p)_{K}^{\text {an }}-\mathcal{U}_{1}(N p)=\mathcal{Z}^{0}(N p)$. This will be done in two steps. The first step is to find a candidate for what the extension of $f$ to $X_{1}(N p)_{K}^{\text {an }}$ should be on $\mathcal{Z}^{0}(N p)$. The second step is to show that this candidate indeed glues to (the Buzzard extension of) $f$. This step uses a general gluing lemma that we prove in this paper, and involves a number of norm estimations on modular forms using the theory of canonical subgroups. The idea of the first part, however, is quite simple (and fun!). Since in the paper this idea is presented in a rather implicit way, we will give a clearer explanation for the benefit of the reader.

To get an idea of how one should define $f$ on $\mathcal{Z}^{0}(N p)$, let us assume that $f$ is classical for the moment, and see how the values of $f$ on $\mathcal{Z}^{0}(N p)$ are related to its values on the complement of $\mathcal{Z}^{0}(N p)$ (where we know $f$ ). We remark that for a modular form $f$ and a test object $(E, i, P)$ we think of $f(E, i, P)$ as an element of $H^{0}\left(E, \Omega_{E}\right)^{\otimes k}$, à la Katz. Assume $(E, i, P)$ is in $\mathcal{Z}^{0}(N p)$. Since $\mathrm{U} f=a f$, we can write

$$
f(E, i, P)=(1 / a p) \sum_{P \notin C_{1}}\left(\mathrm{pr}^{*}\right)^{k} f\left(E / C_{1}, \bar{i}, \bar{P}\right)
$$

where the sum is over the cyclic subgroups $C_{1}$ of order $p=p^{1}$ which do not contain $P$, and pr: $E \rightarrow E / C_{1}$ is the natural projection. By "pr*" we denote the pull-back of oneforms under "pr". One can show that all but one of the test objects appearing on the right hand side of the above formula belong to $\mathcal{Z}^{\infty}(N p)$. The exceptional term corresponds 
to $C=H_{1}$, the first canonical subgroup of $E$. Applying the above formula to $\left(E / H_{1}, \bar{i}, \bar{P}\right)$ we get

$$
f\left(E / H_{1}, \bar{i}, \bar{P}\right)=(1 / a p) \sum_{P \notin C_{2}, H_{1} \subset C_{2}}\left(\operatorname{pr}^{*}\right)^{k} f\left(E / C_{2}, \bar{i}, \bar{P}\right)
$$

where the sum is over the cyclic subgroups $C_{2}$ of order $p^{2}$ which contain $H_{1}$, but not $P$. Combining the two equations we get

$$
f(E, i, P)=(1 / a p) \sum_{P \notin C_{1}, C_{1} \neq H_{1}}\left(\mathrm{pr}^{*}\right)^{k} f\left(E / C_{1}, \bar{i}, \bar{P}\right)+(1 / a p)^{2} \sum_{P \notin C_{2}, H_{1} \subset C_{2}}\left(\mathrm{pr}^{*}\right)^{k} f\left(E / C_{2}, \bar{i}, \bar{P}\right) .
$$

We will repeat this process ad infinitum. At the $n$-th step, we separate the term corresponding to the quotient of $E$ by $H_{n}$, the $n$-th canonical subgroup, and rewrite the term via Equation (11). At the $n$-th step the error term is $(1 / a p)^{n}\left(\mathrm{pr}^{*}\right)^{k} f\left(E / H_{n}, \bar{i}, \bar{P}\right)$. Since on the (good-reduction locus of the) ordinary part $H_{n}$ reduces to the kernel of $\operatorname{Fr}_{p}^{n}$ modulo $p$, one can show that the error term is divisible by $(1 / a p)^{n} p^{n k}=\left(p^{k-1} / a\right)^{n}$. Since $v(a)<k-1$, we see that the error term goes to zero as $n$ goes to infinity. The same estimate can be made on the locus of bad reduction as well (see Corollary 3.5). The result is the following.

Theorem 1.3. Let $f$ be a classical modular form of weight $k$ and level $\Gamma_{1}(N p)$ defined over $K$. Assume that $f$ satisfies $\mathrm{U} f=a f$ with $v(a)<k-1$. Then for $(E, i, P) \in \mathcal{Z}^{0}(N p)$ we have

$$
f(E, i, P)=\sum_{n=1}^{\infty}(1 / a p)^{n}\left(\sum_{C_{n}}\left(\operatorname{pr}^{*}\right)^{k} f\left(E / C_{n}, \bar{i}, \bar{P}\right)\right)
$$

where $C_{n}$ runs through all the cyclic subgroups of $E$ of order $p^{n}$ which contain $H_{n-1}$, are different from $H_{n}$, and do not contain $P$.

Now, let us go back to the assumption that $f$ is overconvergent of slope less than $k-1$. One can show that all the test objects appearing on the right hand side of the above formula belong to $\mathcal{Z}^{\infty}(N p)$, and hence, $f$ is already defined for them. This suggests that the extension of $f$ to $\mathcal{Z}^{0}(N p)$, denoted by $g$, shall be defined via the above series. However this definition will only work on $\mathcal{Z}^{0}(N p)$ where $H_{n}$ exists for all $n$. The rest is to prove that $g$ which is defined on $\mathcal{Z}^{0}(N p)$, will glue to $f$ which is defined on the complement of $\mathcal{Z}^{0}(N p)$. And that will follow from our gluing lemma, as we shall see.

Here goes the structure of this paper. In $\$ 2$ we prove the gluing lemma. In $\$ 3$ we review some background on the theory of modular curves and overconvergent modular forms, and present (a slight generalization of) Buzzard's analytic continuation results. In 4 we prove Theorem 1.2. we construct $g$ described above, and show that the gluing lemma can be applied to glue $f$ and $g$ to produce a classical modular form. To show that the lemma applies, we obtain a variety of norm estimations using the theory of canonical subgroups, and in particular, we prove the boundedness of the Buzzard extension of $f$ on the wide open space $\mathcal{U}_{1}\left(N p^{m}\right)$.

Acknowledgment. We are grateful to Kevin Buzzard and Richard Taylor for their paper [BT] which inspired us to pursue the strategy of analytic continuation. More thanks are due to Buzzard for his paper $[\mathrm{Bu}$, the influence of which on this work is clear. We also thank Robert Coleman and Ofer Gabber for interesting discussions. Finally, we thank the referee for a very close reading of this article, and the many useful suggestions and comments that helped improve the presentation of this manuscript. 


\section{The Gluing Lemma}

In this section we prove a gluing lemma for sections of line bundles on rigid analytic varieties. Let $K$ be a finite extension of $\mathbb{Q}_{p}$ with the ring of integers $\mathcal{O}_{K}$. Let $v$ denote the valuation on $K$ normalized to satisfy $v(p)=1$. For $c \in K$ define $|c|=p^{-v(c)}$. Let $X$ be a reduced flat scheme of finite type over $\mathcal{O}_{K}$. Let $X_{K}$ denote $X \otimes_{\mathcal{O}_{K}} K$. Denote the completion of $X$ along its special fibre by $\tilde{X}$. Note that $\tilde{X}$ is flat and topologically finitely generated over $\mathcal{O}_{K}$ (i.e., an admissible formal scheme over $\mathcal{O}_{K}$ ). Raynaud's functor associates to $\tilde{X}$, its generic fibre $\tilde{X}_{\text {rig }}$, which is a quasi-compact and quasi-separated rigid analytic space over $K$.

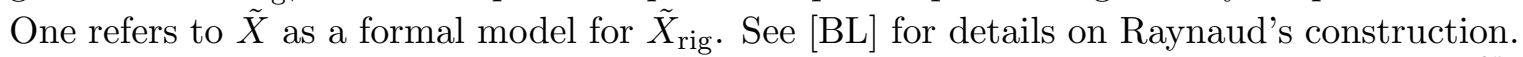
There is also the analytification functor which to $X_{K}$ associates a rigid analytic space $X_{K}^{\text {an }}$. In general, there is an open immersion $\tilde{X}_{\text {rig }} \hookrightarrow X_{K}^{\text {an }}$ which is an isomorphism if $X$ is proper over $\mathcal{O}_{K}$.

Let $M$ be an invertible sheaf on $X$. This induces invertible sheaves $M_{K}, \tilde{M}, \tilde{M}_{\text {rig }}, M_{K}^{\text {an }}$ respectively on $X_{K}, \tilde{X}, \tilde{X}_{\text {rig }}, X_{K}^{\text {an }}$. Let us fix a finite trivialization of $\tilde{M}$ on $\tilde{X}$

$$
\left\{\left(\tilde{U}_{i}=\operatorname{Spf}\left(A_{i}\right), \tilde{\sigma}_{i}: \tilde{M}_{\tilde{U}_{i}} \stackrel{\sim}{\longrightarrow} \mathcal{O}_{\tilde{U}_{i}}\right)\right\}_{i \in I} .
$$

This induces a trivialization for $\tilde{M}_{\text {rig }}$ on $\tilde{X}_{\text {rig }}$ which we denote by $\left\{\left(\mathcal{U}_{i}:=\left(\tilde{U}_{i}\right)_{\text {rig }}, \sigma_{i}\right)\right\}_{i \in I}$.

Definition 2.1. Fix the formal scheme $\tilde{X}$ and the sheaf $\tilde{M}$ on it. Let $x \in \tilde{X}_{\text {rig }}$ be a

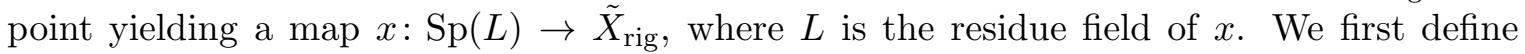
a norm ||$_{x}$ on $H^{0}\left(\operatorname{Sp}(L), x^{*} \tilde{M}_{\text {rig }}\right)$. Denote the formal lifting of $x$ to the formal model by $\tilde{x}: \operatorname{Spf}\left(\mathcal{O}_{L}\right) \rightarrow \tilde{X}$, where $\mathcal{O}_{L}$ is the ring of integers in $L$. Then

$$
H^{0}\left(\operatorname{Sp}(L), x^{*} \tilde{M}_{\text {rig }}\right)=H^{0}\left(\operatorname{Spf}\left(\mathcal{O}_{L}\right), \tilde{x}^{*} \tilde{M}\right) \otimes_{\mathcal{O}_{L}} L
$$

and we define ||$_{x}$ via identifying $H^{0}\left(\operatorname{Spf}\left(\mathcal{O}_{L}\right), \tilde{x}^{*} \tilde{M}\right)$ with $\mathcal{O}_{L}$. Clearly, the definition is independent of the identification. Now, consider an admissible open subset $\mathcal{U} \subset \tilde{X}_{\text {rig }}$, and let $f \in H^{0}\left(\mathcal{U}, \tilde{M}_{\text {rig }}\right)$ and $x \in \mathcal{U}$. We define

$$
|f(x)|:=\left|x^{*} f\right|_{x} .
$$

We also define the norm of $f$ over $\mathcal{U}$ (possibly infinite) to be

$$
|f|_{\mathcal{U}}:=\sup \{|f(x)|: x \in \mathcal{U}\} \text {. }
$$

If $x \in \mathcal{U} \cap \mathcal{U}_{i}$, we can use the identification $H^{0}\left(\operatorname{Spf}\left(\mathcal{O}_{L}\right), \tilde{x}^{*} \tilde{M}\right) \cong \mathcal{O}_{L}$ induced by $\tilde{\sigma}_{i}$ to calculate $|f(x)|$. This shows that

$$
|f|_{\mathcal{U}}=\max _{i \in I}\left\{\left|\sigma_{i}\left(f_{\left.\right|_{\mathcal{U} \cap \mathcal{U}_{i}}}\right)\right|_{\text {sup }}\right\}
$$

where the ||$_{\text {sup }}$ on the right is the usual supremum norm of functions.

Lemma 2.2. If $\mathcal{U}$ is an affinoid subdomain of $\tilde{X}_{\text {rig }}$, then ||$_{\mathcal{U}}$ is a norm on $H^{0}\left(\mathcal{U}, \tilde{M}_{\text {rig }}\right)$ which makes it into a $K$-Banach module.

Proof. First we show that ||$_{\mathcal{U}}$ is finite. Since $\tilde{X}_{\text {rig }}$ is quasi-separated, each $\mathcal{U} \cap \mathcal{U}_{i}$ is quasicompact and hence, it can be covered by finitely many affinoids. Therefore, the supremum norm of functions is finite on each $\mathcal{U} \cap \mathcal{U}_{i}$. Now, the result follows from Equation (4) since $I$ is finite. 
In general ||$_{\text {sup }}$ gives a complete and separated norm on functions on a reduced affinoid. The fact that $\tilde{X}_{\text {rig }}$ is reduced along with Equation (4) implies that ||$_{\mathcal{U}}$ is separated.

For the completeness, note that a Cauchy sequence consisting of elements in $H^{0}\left(\mathcal{U}, \tilde{M}_{\text {rig }}\right)$ produces, for each $i \in I$, a Cauchy sequence of functions on the quasi-compact $\mathcal{U} \cap \mathcal{U}_{i}$. Each of these Cauchy sequences of functions converges, and the limits can be used, via the trivialization in question, to produce a section of $\tilde{M}_{\text {rig }}$ on $\mathcal{U}$ which lies in the limit of the original Cauchy sequence.

Now, we prove our gluing lemma.

Lemma 2.3. (The Gluing Lemma). Let the notation be as above. Let $\mathcal{X} \subset \tilde{X}_{\text {rig }}$ be a smooth affinoid subdomain. Assume that $\mathcal{X}$ is a disjoint union of two admissible opens $\mathcal{X}=$ $\mathcal{Y} \cup \mathcal{Z}$, where $\mathcal{Z}$ is an affinoid. Assume we are given affinoid subdomains of $\mathcal{X}$ denoted by $\mathcal{Z}_{n}$ for $n \geq 1$ with

$$
\mathcal{Z} \subset \mathcal{Z}_{1} \subset \mathcal{Z}_{2} \subset \cdots
$$

and such that $\left\{\mathcal{Y}, \mathcal{Z}_{n}\right\}$ is an admissible cover of $\mathcal{X}$ for each $n$. Assume that we are given two sections

$$
f \in H^{0}\left(\mathcal{Y}, \tilde{M}_{\text {rig }}\right) \quad g \in H^{0}\left(\mathcal{Z}, \tilde{M}_{\text {rig }}\right)
$$

and for each $n \geq 1$, a section $F_{n} \in H^{0}\left(\mathcal{Z}_{n}, \tilde{M}_{\text {rig }}\right)$ such that, as $n \rightarrow \infty$, we have

$$
\left|F_{n}-f\right|_{\mathcal{Y} \cap \mathcal{Z}_{n}} \rightarrow 0 \text { and }\left|F_{n}-g\right|_{\mathcal{Z}} \rightarrow 0 \text {. }
$$

Then $f$ and $g$ glue together to give a global section of $\tilde{M}_{\text {rig }}$ on $\mathcal{X}$. In other words, there is a section of $\tilde{M}_{\text {rig }}$ on $\mathcal{X}$, which restricts to $f$ on $\mathcal{Y}$, and restricts to $g$ on $\mathcal{Z}$.

Proof. Since gluing $f$ and $g$ is a local issue, and in view of Equation (4), we can assume that $\tilde{M}_{\text {rig }}$ restricted to $\mathcal{X}$ is the structure sheaf, and that for a section $f$ (over an open of $\mathcal{X}$ ), and for $x \in \mathcal{X}$, the norm, $|f(x)|$, is the same as the spectral norm for functions. From now on, we will think of $f, g$, and all $F_{n}$ 's as analytic functions, and of all norms as supremum norms.

Let $\check{\mathcal{O}}_{\mathcal{X}}$ denote the subsheaf of $\mathcal{O}_{\mathcal{X}}$ whose sections over an admissible open are those analytic functions with supremum norm less than 1 . It is not difficult to see that the assumptions of the lemma imply that $|f|_{\mathcal{Y}}$ and $|g|_{\mathcal{Z}}$ are finite, and $\left|F_{n}\right|_{\mathcal{Z}_{n}}$ are bounded independently of $n$, and hence, by rescaling, we can assume that $f, g$, and all $F_{n}$ 's are sections of $\check{\mathcal{O}}_{\mathcal{X}}$. We can also assume that

$$
\left|F_{n}-f\right|_{\mathcal{Y} \cap \mathcal{Z}_{n}}<(1 / p)^{n} \text { and }\left|F_{n}-g\right|_{\mathcal{Z}}<(1 / p)^{n} .
$$

Let $\check{\mathcal{O}}_{\mathcal{X}} / p^{n} \check{\mathcal{O}}_{\mathcal{X}}$ denote the quotient sheaf. Then the reduction of $f$ (resp., $F_{n}$ ) modulo $p^{n}$ is a section of $\check{\mathcal{O}}_{\mathcal{X}} / p^{n} \check{\mathcal{O}}_{\mathcal{X}}$ on $\mathcal{Y}$ (resp., $\mathcal{Z}_{n}$ ) and by Equation (5) they agree over $\mathcal{Y} \cap \mathcal{Z}_{n}$. This implies that they glue together to give a section $h_{n}$ of $\check{\mathcal{O}}_{\mathcal{X}} / p^{n} \check{\mathcal{O}}_{\mathcal{X}}$ over $\mathcal{X}$. The conditions of the lemma imply that $h_{n}$ 's are compatible in the sense that they give an element of the inverse limit

$$
\varliminf_{n} \lim _{\mathcal{X}} / p^{n} \check{\mathcal{O}}_{\mathcal{X}}(\mathcal{X}) \text {. }
$$

Theorem 2 of $\mathrm{Ba}$ tells us that, since $\mathcal{X}$ is a smooth affinoid, there exists a non-zero $c \in K$ with $|c| \leq 1$ such that $c H^{1}\left(\mathcal{X}, \check{\mathcal{O}}_{\mathcal{X}}\right)=0$. This implies that

$$
c h_{n} \in \check{\mathcal{O}}_{\mathcal{X}}(\mathcal{X}) / p^{n} \check{\mathcal{O}}_{\mathcal{X}}(\mathcal{X}) \subset \underline{\check{\mathcal{O}}_{\mathcal{X}} / p^{n} \check{\mathcal{O}}_{\mathcal{X}}}(\mathcal{X}),
$$


and hence $\left\{c h_{n}\right\}$ defines an element of $\lim _{n} \check{\mathcal{O}}_{\mathcal{X}}(\mathcal{X}) / p^{n} \check{\mathcal{O}}_{\mathcal{X}}(\mathcal{X})=\check{\mathcal{O}}_{\mathcal{X}}(\mathcal{X})$ (since $\check{\mathcal{O}}_{\mathcal{X}}(\mathcal{X})$ is $p$-adically complete). We define $h$ to be the section of $\mathcal{O}_{\mathcal{X}}$ over $\mathcal{X}$ obtained by dividing the above section by $c$.

By definition of $h$, we have $c h_{\mid \mathcal{Y}}-c f \in p^{n} \check{\mathcal{O}}_{\mathcal{X}}(\mathcal{Y})$ for all $n \geq 1$, and hence, we find that $h$ restricts to $f$ on $\mathcal{Y}$. Similarly, we find that $c h_{\mid \mathcal{Z}}-c g=\left(c h_{\mid \mathcal{Z}}-c F_{\left.n\right|_{\mathcal{Z}}}\right)+\left(c F_{\left.n\right|_{\mathcal{Z}}}-c g\right) \in p^{n} \check{\mathcal{O}}_{\mathcal{X}}(\mathcal{Z})$ for all $n \geq 1$ which implies that $h$ restricts to $g$ over $\mathcal{Z}$. We are done.

Remark 2.4. The smoothness hypothesis can be removed at the expense of making the lemma more technically involved. Since in the applications to overconvergent modular forms the smoothness can be afforded we have chosen to make this assumption. We are grateful to Ofer Gabber for pointing to us Bartenwerfer's result on the cohomology of the sheaf $\check{\mathcal{O}}_{\mathcal{X}}$.

\section{OVERCONVERGENT MODULAR FORMS}

We review some background on the theory of overconvergent modular forms. For more details we refer the reader to consult [KM], $\mathrm{Bu}]$.

3.1. Modular curves and modular forms. Our main reference is [KM]. Let $N>4$ be an integer and $p$ be a prime number such that $(p, N)=1$. By $X_{1}(N)$ we denote the smooth and proper modular curve over $\mathbb{Z}[1 / N]$ whose non-cuspidal part, denoted $Y_{1}(N)$, classifies pairs $(E, i)$, where $E$ is an elliptic curve over a $\mathbb{Z}[1 / N]$-scheme, and $i: \mu_{N} \rightarrow E$ is an embedding of the constant group scheme $\mu_{N}$ in $E$. For any $\mathbb{Z}[1 / N]$-algebra $R$, we let $X_{1}(N)_{R}$ denote the base extension of $X_{1}(N)$ to $R$. Denote by $E_{1}(N)$ the universal family of elliptic curves over $Y_{1}(N)$. There is a well-known locally free sheaf of rank one, $\underline{\omega}=\underline{\omega}_{X_{1}(N)}$, whose restriction to $Y_{1}(N)$ is the push-forward of $\Omega_{E_{1}(N) / Y_{1}(N)}^{1}$ under the natural map from $E_{1}(N)$ to $Y_{1}(N)$. The space of modular forms of weight $k \in \mathbb{Z}_{>0}$ and level $\Gamma_{1}(N)$ over $R$, denoted by $M_{k}\left(\Gamma_{1}(N), R\right)$, is $H^{0}\left(X_{1}(N)_{R}, \underline{\omega}^{k}\right)$.

Let $X_{1}(N, p)$ denote the flat and proper modular curve over $\mathbb{Z}[1 / N]$ whose non-cuspidal part, $Y_{1}(N, p)$, classifies triples $(E, i, C)$, where $(E, i)$ is as above, and $C$ is a finite flat subgroup of $E$ of order $p$. For any $\mathbb{Z}[1 / N]$-algebra, $R$, we let $X_{1}(N, p)_{R}$ denote the base extension of $X_{1}(N, p)$ to $R$. As in the above, there is a universal elliptic curve $E_{1}(N, p)$ over $Y_{1}(N, p)$, and a locally free sheaf $\underline{\omega}=\underline{\omega}_{X_{1}(N, p)}$ over $X_{1}(N, p)$ whose restriction to $Y_{1}(N, p)$ is the push-forward of $\Omega_{E_{1}(N, p) / Y_{1}(N, p)}^{1}$. If we let $\pi_{1}: X_{1}(N, p) \rightarrow X_{1}(N)$ denote the degeneracy map which (on the non-cuspidal part) forgets the subgroup of order $p$, then one has

$$
\underline{\omega}_{X_{1}(N, p)}=\pi_{1}^{*} \underline{\omega}_{X_{1}(N)} .
$$

If $p$ is invertible in $R$, we define the space of modular forms of weight $k \in \mathbb{Z}_{\geq 0}$ and level $\Gamma_{1}(N) \cap \Gamma_{0}(p)$ over $R$, denoted by $M_{k}\left(\Gamma_{1}(N) \cap \Gamma_{0}(p), R\right)$, to be $H^{0}\left(X_{1}(N, p)_{R}, \underline{\omega}^{k}\right)$.

Assume $m>0$. Let $X_{1}\left(N p^{m}\right)$ denote the proper and flat modular curve over $\mathbb{Z}[1 / N]$, the non-cuspidal part of which, denoted $Y_{1}\left(N p^{m}\right)$, classifies triples $(E, i, P)$, where $(E, i)$ is as above, and $P$ is a point of exact order $p^{m}$. For any $\mathbb{Z}[1 / N]$-algebra $R$, we let $X_{1}\left(N p^{m}\right)_{R}$ denote the base extension of $X_{1}\left(N p^{m}\right)$ to $R$. If $p$ is invertible in $R$, then $X_{1}\left(N p^{m}\right)_{R}$ is smooth. As in the case of $X_{1}(N)$, there a universal family of elliptic curves $E_{1}\left(N p^{m}\right)$ over $Y_{1}\left(N p^{m}\right)$, and a locally free sheaf of rank one, $\underline{\omega}=\underline{\omega}_{X_{1}\left(N p^{m}\right)}$, whose restriction to $Y_{1}\left(N p^{m}\right)$ is the pushforward of $\Omega_{E_{1}\left(N p^{m}\right) / Y_{1}\left(N p^{m}\right)}^{1}$. The pullback of $\underline{\omega}_{X_{1}(N)}$ under the natural map from $X_{1}\left(N p^{m}\right)$ to $X_{1}(N)$ which (on the non-cuspidal part) forgets the point of order $p^{m}$ is $\underline{\omega}_{X_{1}\left(N p^{m}\right)}$. When $p$ is invertible in $R$, the space of modular forms of weight $k \in \mathbb{Z}_{\geq 0}$ and level $\Gamma_{1}\left(N p^{m}\right)$ over $R$, denoted by $M_{k}\left(\Gamma_{1}\left(N p^{m}\right), R\right)$, is $H^{0}\left(X_{1}\left(N p^{m}\right)_{R}, \underline{\omega}^{k}\right)$. 
3.2. Overconvergent modular forms. Let $K / \mathbb{Q}_{p}$ be a finite extension of $\mathbb{Q}_{p}$ with valuation $v$ normalized so that $v(p)=1$, and with the corresponding norm ||$=(1 / p)^{v}$. Let $\mathcal{O}_{K}$ denote the ring of integers, and $\kappa$ the residue field.

Let $X$ denote any of the modular curves described above. There is a rigid analytic space $X_{K}^{\text {an }}$ associated to the modular curve $X_{K}$ over $K$. We will denote the analytification of the sheaf $\underline{\omega}$ again by $\underline{\omega}$. Let $\mathcal{U}$ be an admissible open subset of $X_{K}^{\text {an }}$, and let $f \in H^{0}\left(\mathcal{U}, \underline{\omega}^{k}\right)$. If $x \in \mathcal{U}$ is a point, we define $|f(x)|$ as in Definition [2.1] using the admissible formal scheme $\tilde{X}_{\mathcal{O}_{K}}$, which is the completion of $X_{\mathcal{O}_{K}}$ along its special fibre, and the induced invertible sheaf $\underline{\omega}^{k}$ on $\tilde{X}_{\mathcal{O}_{K}}$. When $p>3$, the Eisenstein series $E_{p-1}$ is a modular form of weight $p-1$ which lifts the Hasse invariant from characteristic $p$. For $x \in X_{K}^{\text {an }}$ we define the "measure of supersingularity" of $x$ to be $\left|E_{p-1}(x)\right|$. In general, and to include the cases $p=2,3$, the measure of supersingularity of $x$ can essentially be defined via the norm of a parameter on the completion of $X_{1}(N)_{\mathcal{O}_{K}}$ at the reduction of the "point" which induces the prime-to- $p$ part of $x$. This coincides with $\left|E_{p-1}(x)\right|$ when $p>3$ and $\left|E_{p-1}(x)\right|<1$. For details we refer the reader to $\S 3$ of $[\mathrm{Bu}$ ] (where things are defined using valuation rather than norm). In this paper, we always use $\left|E_{p-1}(x)\right|$ for the measure of supersingularity, and keep in mind its generalized sense when $p=2,3$.

We will define various rigid analytic subdomains of $X_{K}^{\text {an }}$. Let $1 / p<r \in\left|\mathbb{C}_{p}\right|$. Let $X_{1}(N)_{\bar{K}}^{\geq r}$ be the affinoid subdomain of $X_{1}(N)_{K}^{\text {an }}$ whose points are given by

$$
\left\{x \in X_{1}(N)_{K}^{\text {an }}:\left|E_{p-1}(x)\right| \geq r\right\} .
$$

The space of overconvergent modular forms of weight $k$ and level $\Gamma_{1}(N)$ over $K$ is

$$
M_{k}^{\dagger}(N, K)=\underset{p^{-p /(p+1)}<r \rightarrow 1^{-}}{\lim _{\longrightarrow}} H^{0}\left(X_{1}(N)_{\bar{K}}^{\geq r}, \underline{\omega}^{k}\right) .
$$

If $(E, i) / L$ is a point on $X_{1}(N)_{K}^{\text {an }}$ such that $\left|E_{p-1}(E, i)\right|>p^{-p /(p+1)}$ (i.e., if $(E, i)$ is not too supersingular), then $E[p]$ has a canonical subgroup $H_{1}=H_{1}(E)$ of order $p$, which is defined over $\mathcal{O}_{L}$ if $E$ has good reduction, and serves as a canonical lifting of the kernel of Frobenius. With more restriction on the supersingularity of $(E, i)$ we can define canonical subgroups of higher order. For example, if $\left|E_{p-1}(E, i)\right|>p^{-1 /(p+1)}$, one can show that $\left|E_{p-1}\left(E / H_{1}(E), \bar{i}\right)\right|>p^{-p /(p+1)}$, and hence $E / H_{1}(E)$ has a canonical subgroup of order $p$. The inverse image of this subgroup in $E$ is a cyclic subgroup of order $p^{2}$ of $E$, denoted by $H_{2}(E)$, which contains $H_{1}(E)$. Similarly if $\left|E_{p-1}(E, i)\right|>p^{-p^{2-n} /(p+1)}$, then $E$ has a cyclic canonical subgroup of order $p^{n}$, denoted by $H_{n}(E)$, and one has

$$
H_{1}(E) \subset H_{2}(E) \subset \ldots \subset H_{n}(E) .
$$

For a detailed analysis of canonical subgroups and the history of the subject see $\S 3$ of $\mathrm{Bu}$.

Let $r>p^{-p /(p+1)}$. The map which (on the non-cuspidal part) sends $(E, i)$ to $\left(E, i, H_{1}\right)$ provides a section for the forgetful morphism $\pi_{1}^{\text {an }}: X_{1}(N, p)_{K}^{\text {an }} \rightarrow X_{1}(N)_{K}^{\text {an }}$ over $X_{1}(N)_{K}^{\geq r}$, and hence, gives an isomorphism between $X_{1}(N)_{K}^{\geq r}$ and its image in $X_{1}(N, p)_{K}^{\text {an }}$. The image is denoted by $X_{1}(N, p)_{\bar{K}}^{\geq r}$, and is the connected component of the cusp $\infty$ in the affinoid subdomain of $X_{1}(N, p)_{K}$ defined by $\left|E_{p-1}(x)\right| \geq r$. Its non-cuspidal points consist of all $(E, i, C)$ such that $\left|E_{p-1}(E, i)\right| \geq r$ and $C=H_{1}(E)$. This shows that there is an inclusion

$$
H^{0}\left(X_{1}(N, p)_{K}^{\text {an }}, \underline{\omega}^{k}\right) \hookrightarrow M_{k}^{\dagger}(N, K)
$$


and we call the modular forms in the image classical (over $\left.X_{1}(N, p)_{K}\right)$. Indeed, since $X_{1}(N, p)$ is proper over $\mathbb{Z}[1 / N]$, we know that the analytification map

$$
H^{0}\left(X_{1}(N, p)_{K}, \underline{\omega}^{k}\right) \stackrel{\text { an }}{\longrightarrow} H^{0}\left(X_{1}(N, p)_{K}^{\text {an }}, \underline{\omega}^{k}\right)
$$

is an isomorphism.

We define these concepts for level $\Gamma_{1}\left(N p^{m}\right)$ where $m>0$. Consider the map

$$
X_{1}\left(N p^{m}\right)_{K} \rightarrow X_{1}(N, p)_{K}
$$

which on the non-cuspidal part sends $(E, i, P)$ to $(E /\langle p P\rangle, \bar{i}, \bar{P})$. Let $r$ be an element of $\left|\mathbb{C}_{p}\right|$ such that $r>p^{-p^{2-m} /(p+1)}$. Define $X_{1}\left(N p^{m}\right) \frac{\geq r}{K}$ to be the inverse image of $X_{1}(N, p) \frac{\geq r^{p^{m-1}}}{K}$ under this map. The non-cuspidal part of $X_{1}\left(N p^{m}\right)_{\bar{K}}^{\geq r}$ consists of all $(E, i, P)$ such that $\left|E_{p-1}(E, i)\right| \geq r$, and $H_{m}(E)=\langle P\rangle$. The space of overconvergent modular forms of weight $k$ and level $\Gamma_{1}\left(N p^{m}\right)$ over $K$ is

$$
M_{k}^{\dagger}\left(N p^{m}, K\right)=\underset{(1 / p)^{p^{2-m}}}{\lim _{/(p+1)}<r \rightarrow 1^{-}} H^{0}\left(X_{1}\left(N p^{m}\right)_{K}^{\geq r}, \underline{\omega}^{k}\right) .
$$

There is, therefore, an inclusion

$$
H^{0}\left(X_{1}\left(N p^{m}\right)_{K}^{\text {an }}, \underline{\omega}^{k}\right) \hookrightarrow M_{k}^{\dagger}\left(N p^{m}, K\right) .
$$

An overconvergent modular form in $M_{k}^{\dagger}\left(N p^{m}, K\right)$ is said to be classical (over $X_{1}\left(N p^{m}\right)_{K}$ ) if it is in the image of the above inclusion, i.e., if it can be extended to a section of $\underline{\omega}^{k}$ on $X_{1}\left(N p^{m}\right)_{K}^{\text {an }}$.

By the $q$-expansion of an overconvergent modular form we mean the $q$-expansion at the cusp $\infty$. There is a Hecke operator $\mathrm{U}_{p}=\mathrm{U}$ acting on $M_{k}^{\dagger}\left(N p^{m}, K\right)$ which on the $q$-expansions has the effect

$$
\mathrm{U}\left(\sum a_{n} q^{n}\right)=\sum a_{n p} q^{n}
$$

By a generalized eigenform of $\mathrm{U}$ we mean an overconvergent modular form $f$, for which there is $R(x) \in K[x]$ such that $R(\mathrm{U}) f=0$. We say that $f$ has slope $\alpha \in \mathbb{Q}$, if all the roots of $R(x)$ in $\mathbb{C}_{p}$ have valuation $\alpha$. For instance, the slope of a U-eigenform is the valuation of its eigenvalue. One knows that the slope of a classical U-eigenform of level $\Gamma_{1}(N p)$ and weight $k$ is at most $k-1$.

In this paper we think about modular forms as Katz does in $\mathrm{Ka}$, albeit in a rigid analytic sense. In particular, if $f$ is a modular form of weight $k$ and level $\Gamma$ defined over an admissible open $\mathcal{U}$ of the corresponding modular curve, and $(E, \gamma) \in \mathcal{U}$ is an elliptic curve with level $\Gamma$ structure, then $f(E, \gamma) \in H^{0}\left(E, \Omega_{E}\right)^{\otimes k}$.

3.3. Buzzard's analytic continuation results. In this subsection, we will recall the analytic continuation results obtained by Buzzard in his paper $\left[\mathrm{Bu}\right.$. Let $\mathcal{Z}^{0}(N, p)$ be the connected component of the cusp 0 in the ordinary part of $X_{1}(N, p)_{K}^{\text {an }}$. Let $\mathcal{U}_{1}(N, p)$ be the complement of $\mathcal{Z}^{0}(N, p)$. It is an admissible open in $X_{1}(N, p)_{K}^{\text {an }}$ whose non-cuspidal points consist of $(E, i, C)$ such that either $E$ has supersingular reduction, or $E$ has ordinary reduction and $C=H_{1}(E)$. This is denoted by $\mathcal{W}_{0}(p)$ in $[\mathrm{Bu}]$.

Assume $m>0$. There is a map from $X_{1}\left(N p^{m}\right)_{K}$ to $X_{1}(N, p)_{K}$ which on the non-cuspidal part sends $(E, i, P)$ to $\left(E, i,\left\langle p^{m-1} P\right\rangle\right)$. Let $\mathcal{U}_{1}\left(N p^{m}\right)$ denote the inverse image of $\mathcal{U}_{1}(N, p)$ under this map. It is an admissible open of $X_{1}\left(N p^{m}\right)_{K}$ whose non-cuspidal points consist of all $(E, i, P)$ such that either $E$ has supersingular reduction, or $E$ is of ordinary reduction and 
the subgroup generated by $P$ contains $H_{1}(E)$. In the case $m=1$, Buzzard denotes $\mathcal{U}_{1}(N p)$ by $\mathcal{W}_{1}(p)$.

Buzzard shows that any overconvergent U-eigenform with non-zero eigenvalue can be extended to $\mathcal{U}_{1}\left(N p^{m}\right)$. We will use a generalization of this fact which can be proved by exactly the same method.

Theorem 3.1. Let $f$ be an overconvergent modular form of level $\Gamma_{1}\left(N p^{m}\right)$ over $K$. Assume that for some $R(x) \in K[x]$ with $R(0) \neq 0$ we can extend $R(\mathrm{U}) f$ to $\mathcal{U}_{1}\left(N p^{m}\right)$. Then $f$ can be extended to $\mathcal{U}_{1}\left(N p^{m}\right)$. The same statement is valid in level $\Gamma_{1}(N) \cap \Gamma_{0}(p)$.

Proof. Let $R(0)=-a$, and $R_{0}(x)=R(x)+a$. Assume $R(\mathrm{U}) f=F$ which is defined over $\mathcal{U}_{1}\left(N p^{m}\right)$. The same method as in $\mathrm{Bu}$ ] works: only at the $n$-th step instead of considering $\mathrm{U}^{n} f / a^{n}$ as the partial analytic continuation, one should take

$$
R_{0}(\mathrm{U})^{n} f / a^{n}-R_{0}(\mathrm{U})^{n-1} F / a^{n}-\ldots-R_{0}(\mathrm{U}) F / a^{2}-F / a .
$$

One just needs to notice that $Q(\mathrm{U}) F$ is defined over $\mathcal{U}_{1}\left(N p^{m}\right)$ for any $Q(x) \in K[x]$ satisfying $Q(0)=0$. A similar argument works over $X_{1}(N, p)_{K}$.

3.4. Some notation and a lemma. Fix once and for all $t \in\left|\mathbb{C}_{p}\right|$ with $p^{-p /(p+1)}<t<1$. The region in $X_{1}(N, p)_{K}^{\text {an }}$ where $\left|E_{p-1}\right| \geq t$ has two connected components: the connected component of the cusp $\infty$, and the connected component of the cusp 0 which we denote by $\mathcal{V}_{1}(N, p)$. If $(E, i, C)$ is a non-cuspidal point of $X_{1}(N, p)_{K}^{\text {an }}$, then it lies in $\mathcal{V}_{1}(N, p)$ iff $\left|E_{p-1}(E, i)\right| \geq t$ and $C \neq H_{1}(E)$. This is an affinoid subdomain of $X_{1}(N, p)_{K}^{\text {an }}$, and

$$
\left\{\mathcal{V}_{1}(N, p), \mathcal{U}_{1}(N, p)\right\}
$$

is an admissible covering of $X_{1}(N, p)_{K}^{\text {an }}$. Assume $m>0$. Let

$$
\phi: X_{1}\left(N p^{m}\right)_{K}^{\text {an }} \rightarrow X_{1}(N, p)_{K}^{\text {an }}
$$

denote the map which sends $(E, i, P)$ to $\left(E, i,\left\langle p^{m-1} P\right\rangle\right)$. Define $\mathcal{V}_{1}\left(N p^{m}\right)=\phi^{-1} \mathcal{V}_{1}(N, p)$. Its non-cuspidal points consist of all $(E, i, P)$ such that $\left|E_{p-1}(E, i)\right| \geq t$ and the subgroup generated by $P$ intersects $H_{1}(E)$ trivially. Since $\mathcal{U}_{1}\left(N p^{m}\right)=\phi^{-1} \mathcal{U}_{1}(N, p)$, we know that

$$
\left\{\mathcal{V}_{1}\left(N p^{m}\right), \mathcal{U}_{1}\left(N p^{m}\right)\right\}
$$

is an admissible covering of $X_{1}\left(N p^{m}\right)_{K}^{\text {an }}$. We will use these admissible coverings in 4 when we deal with classicality of overconvergent modular forms.

Let us fix the level $\Gamma_{1}\left(N p^{m}\right)$ with $m>0$. For simplicity, we denote $X_{1}\left(N p^{m}\right)_{K}^{\text {an }}$ by $X_{K}^{\text {an }}$, and $\mathcal{V}_{1}\left(N p^{m}\right)$ by $\mathcal{V}$. For any closed, open, or half-open subinterval $I$ of $[0,1]$ (with endpoints in $\left.\left|\mathbb{C}_{p}\right|\right)$, we define a corresponding subdomain $\mathcal{V} I$ where $\left|E_{p-1}\right|$ falls within that interval. For example $\mathcal{V}[r, s)$ denotes the part of $\mathcal{V}$ where $r \leq\left|E_{p-1}(x)\right|<s$. If $I$ is a closed interval, then $\mathcal{V} I$ is an affinoid. For any interval $I$, let $\mathcal{E} I$ denote the universal elliptic curve over $\mathcal{V} I-$ $\{$ cusps $\}$, and $\mathcal{H}_{1} I$ denote the canonical subgroup of $\mathcal{E} I$. By $I^{p^{n}}$ we denote the interval obtained by raising $I$ to the power $p^{n}$.

Definition 3.2. Let $r \in\left|\mathbb{C}_{p}\right|$ satisfy $t \leq r \leq 1$. Let $I$ be either $[r, 1]$ or $[r, 1)$. Let

$$
\tau: \mathcal{V} I^{1 / p} \rightarrow \mathcal{V} I
$$

be the map whose effect on the non-cuspidal points is given by

$$
\tau(E, i, P)=\left(E / H_{1}, \bar{i}, \bar{P}\right) .
$$

Here $H_{1}$ is the canonical subgroup of $E$ of order $p, \bar{i}$ is the induced level $\Gamma_{1}(N)$ structure on $E / H_{1}$, and $\bar{P}$ is the image of $P$ in $E / H_{1}$. The pullback of $\underline{\omega}_{\left.\right|_{\mathcal{V}}}$ under $\tau$ is an invertible 
sheaf $\underline{\omega}^{\prime}$ on $\mathcal{V} I^{1 / p}$ whose restriction to the non-cuspidal part is $\underline{\omega}\left(\mathcal{E} I^{1 / p} / \mathcal{H}_{1} I^{1 / p}\right) /\left(\mathcal{V} I^{1 / p}-\{c u s p s\}\right)$. Around a cusp in $\mathcal{V}$ the map $\tau$ is given by $q \mapsto q^{p}$, and hence, the pullback under $\tau$ of the canonical differential form on Tate $(q)$, i.e., $d z / z$, is the canonical differential form on $\operatorname{Tate}(q) / H_{1}(\operatorname{Tate}(q)) \cong \operatorname{Tate}\left(q^{p}\right)$, i.e., $d z^{p} / z^{p}$. This implies that $\underline{\omega}^{\prime}$ is the canonical extension (à la Katz-Mazur) of $\underline{\omega}_{\left(\mathcal{E} I^{1 / p} / \mathcal{H}_{1} I^{1 / p}\right) /\left(\mathcal{V} I^{1 / p}-\{\text { cusps }\}\right)}$ to $\mathcal{V} I^{1 / p}$. Therefore, there is a morphism of sheaves on $\mathcal{V} I^{1 / p}$

$$
\left(\mathrm{pr}^{*}\right)^{k}:\left(\underline{\omega}^{\prime}\right)^{k} \rightarrow\left(\underline{\omega}_{\mathcal{V}_{I^{1 / p}}}\right)^{k}
$$

which on the non-cuspidal part is induced by pulling back differential forms under the natural projection pr: $\mathcal{E} I^{1 / p} \rightarrow \mathcal{E} I^{1 / p} / \mathcal{H}_{1} I^{1 / p}$. Let $f$ be a section of $\underline{\omega}^{k}$ on $\mathcal{V} I$. We define $f^{\tau} \in$ $H^{0}\left(\mathcal{V} I^{1 / p}, \underline{\omega}^{k}\right)$ by

$$
f^{\tau}:=p^{-k}\left(\mathrm{pr}^{*}\right)^{k} \tau^{*} f
$$

Hence one has

$$
f^{\tau}(E, i, P)=p^{-k}\left(\mathrm{pr}^{*}\right)^{k} f\left(E / H_{1}, \bar{i}, \bar{P}\right)
$$

where pr: $E \rightarrow E / H_{1}$ is the natural projection. All these can be defined over $X_{1}(N, p)$ as well, in exactly the same way.

In the final part of this section, we prove a lemma which will be used in the proof of Theorem 4.1. Let $Z_{\mathbb{Z}_{p}}$ denote the affine subscheme of $X_{1}(N)_{\mathbb{Z}_{p}}$ where $E_{p-1}$ is invertible. When $p=2$ or 3 , instead of $E_{p-1}$, we can use $E_{4}$. The open non-cuspidal subscheme of $Z_{\mathbb{Z}_{p}}$ represents the functor which to every $\mathbb{Z}_{p}$-algebra, $R$, associates an elliptic curve over $R$ for which $E_{p-1}$ (or $E_{4}$ when $p=2,3$ ) is invertible, with a level $\Gamma_{1}(N)$-structure. For simplicity we denote by $Z$ the base extension of $Z_{\mathbb{Z}_{p}}$ to $\mathcal{O}_{K}$. The formal completion of $Z$ along its special fibre, $\tilde{Z}$, is a formal model for $\mathcal{Z}$, the locus of ordinary reduction in $X_{1}(N)_{K}^{\text {an }}$. Dividing by the canonical subgroup on the non-cuspidal locus induces a map

$$
\sigma_{n}: Z \otimes\left(\mathcal{O}_{K} / p^{n}\right) \rightarrow Z \otimes\left(\mathcal{O}_{K} / p^{n}\right)
$$

for each $n \geq 1$, and by passing to the limit, a map $\tilde{\sigma}: \tilde{Z} \rightarrow \tilde{Z}$. This, in turn, induces the Frobenius morphism $\sigma: \mathcal{Z} \rightarrow \mathcal{Z}$. The sheaf $\underline{\omega}$ on $X_{1}(N)$ provides invertible sheaves on $\tilde{Z}$ and $\mathcal{Z}$ which we again denote by $\underline{\omega}$. Over $\tilde{Z}$, we set $\underline{\omega}^{\prime}:=\tilde{\sigma}^{*} \underline{\omega}$; similarly, over $\mathcal{Z}$, we set $\underline{\omega}^{\prime}:=\sigma^{*} \underline{\omega}$. One can show that in each case $\underline{\omega}^{\prime}$ is the canonical extension to the cusps of the sheaf of invariant differentials of the quotient of the universal elliptic curve by its canonical subgroup. There is, therefore, a map $\mathrm{pr}^{*}: \underline{\omega}^{\prime} \rightarrow \underline{\omega}$ on $\tilde{Z}$ (and also $\mathcal{Z}$ ). Note that all the above constructions are indeed defined over $\mathbb{Z}_{p}$ in the case of $\tilde{Z}$ (and serve as formal models of the corresponding constructions in the case of $\mathcal{Z}$ ). The natural forgetful map $X_{1}\left(N p^{m}\right)_{K}^{\text {an }} \rightarrow X_{1}(N)_{K}^{\text {an }}$ induces a map $\pi: \mathcal{V}[1,1] \rightarrow \mathcal{Z}$ such that $\pi^{*} \underline{\omega}=\underline{\omega}$ and $\pi^{*} \underline{\omega}^{\prime}=\underline{\omega}^{\prime}$. We also have

$$
\sigma \circ \pi=\pi \circ \tau
$$

and

$$
\pi^{*} \circ \mathrm{pr}^{*}=\mathrm{pr}^{*} \circ \pi^{*}
$$

where the pr* on the left is as above, and the one on the right is as in Definition 3.2 
Lemma 3.3. Let $h \in H^{0}\left(\mathcal{V} I, \underline{\omega}^{k}\right)$, where $I$ is either $[r, 1)$ or $[r, 1]$, and $t \leq r \leq 1$. For any point $x \in \mathcal{V} I^{1 / p}$ we have

$$
\left|h^{\tau}(x)\right| \leq|h(\tau x)|\left|E_{p-1}(x)\right|^{-k} .
$$

Proof. Let $x: \operatorname{Sp}(L) \rightarrow \mathcal{V} I^{1 / p}$ denote a point. The map corresponding to the point $\tau x$ is given by $\tau x: \operatorname{Sp}(L) \stackrel{x}{\longrightarrow} \mathcal{V} I^{1 / p} \stackrel{\tau}{\longrightarrow} \mathcal{V} I$. It is enough to show that

$$
|h(\tau x)| \leq 1 \Rightarrow\left|h^{\tau}(x)\right| \leq\left|E_{p-1}(x)\right|^{-k} .
$$

Let $\tilde{X}$ denote the formal completion of $X_{1}\left(N p^{m}\right)_{\mathcal{O}_{K}}$ along its special fibre. Then $\tilde{X}_{\text {rig }}=$ $X_{1}\left(N p^{m}\right)_{K}^{\text {an }}$. Let $\tilde{x}: \operatorname{Spf}\left(\mathcal{O}_{L}\right) \rightarrow \tilde{X}$ be the formal lifting of $x: \operatorname{Sp}(L) \stackrel{x}{\longrightarrow} \mathcal{V} I^{1 / p} \rightarrow X_{1}\left(N p^{m}\right)_{K}^{\text {an }}$. Similarly let $\tilde{\tau x}$ denote the formal lifting of $\tau x$. We have

$$
x^{*} h^{\tau}=x^{*}\left(p^{-k}\left(\operatorname{pr}^{*}\right)^{k} \tau^{*} h\right)=p^{-k}\left(\operatorname{pr}_{x}^{*}\right)^{k}(\tau x)^{*} h
$$

where $\left(\operatorname{pr}^{*}\right)^{k}: H^{0}\left(\mathcal{V} I^{1 / p},\left(\underline{\omega}^{\prime}\right)^{k}\right) \rightarrow H^{0}\left(\mathcal{V} I^{1 / p}, \underline{\omega}^{k}\right)$ is as in Definition 3.2 and

$$
\left(\operatorname{pr}_{x}^{*}\right)^{k}: H^{0}\left(\operatorname{Sp}(L), x^{*}\left(\underline{\omega}^{\prime}\right)^{k}=(\tau x)^{*} \underline{\omega}^{k}\right) \rightarrow H^{0}\left(\operatorname{Sp}(L), x^{*} \underline{\omega}^{k}\right)
$$

is the specialization of $\left(\mathrm{pr}^{*}\right)^{k}$ to $x$, and satisfies $x^{*}\left(\mathrm{pr}^{*}\right)^{k}=\left(\mathrm{pr}_{x}^{*}\right)^{k} x^{*}$. We claim that it suffices to prove

$$
\left.\operatorname{pr}_{x}^{*}\left(H^{0}\left(\operatorname{Spf}\left(\mathcal{O}_{L}\right),(\tilde{\tau} x)^{*} \underline{\omega}\right)\right) \subseteq\left(p / E_{p-1}(x)\right) H^{0}\left(\operatorname{Spf}\left(\mathcal{O}_{L}\right), \tilde{x}^{*} \underline{\omega}\right)\right)
$$

where $E_{p-1}(x)$ is any element of $L$ of norm $\left|E_{p-1}(x)\right|$. The reason is that

$$
|h(\tau x)| \leq 1 \Rightarrow\left|(\tau x)^{*} h\right|_{\tau x} \leq 1 \Rightarrow(\tau x)^{*} h \in H^{0}\left(\operatorname{Spf}\left(\mathcal{O}_{L}\right),(\tilde{\tau x})^{*} \underline{\omega}^{k}\right)
$$

which in conjunction with (8) would give

$$
\left.x^{*} h^{\tau}=p^{-k}\left(\operatorname{pr}_{x}^{*}\right)^{k}(\tau x)^{*} h \in E_{p-1}(x)^{-k} H^{0}\left(\operatorname{Spf}\left(\mathcal{O}_{L}\right), \tilde{x}^{*} \underline{\omega}^{k}\right)\right)
$$

which means that $\left|h^{\tau}(x)\right|=\left|x^{*} h^{\tau}\right|_{x} \leq\left|E_{p-1}(x)\right|^{-k}$ as desired. In what follows we prove (8).

First assume that $x$ corresponds to an elliptic curve (with level structure) $\underline{E} / L$ which is supersingular (or more generally of good reduction). By the assumptions in the lemma, $E$ has a canonical subgroup $H=H_{1}$. The map $\operatorname{pr}_{x}^{*}$ can be interpreted as

$$
\operatorname{pr}_{E}^{*}: H^{0}\left(E / H, \Omega_{E / H}\right) \rightarrow H^{0}\left(E, \Omega_{E}\right)
$$

where $\operatorname{pr}_{E}: E \rightarrow E / H$ is the natural projection. Let $\mathbf{E}$ be the integral model of $E$ over $\mathcal{O}_{L}$, and $\mathbf{H}$ the canonical subgroup of $\mathbf{E}$. Let $\operatorname{pr}_{\mathbf{E}}: \mathbf{E} \rightarrow \mathbf{E} / \mathbf{H}$ denote the projection. To prove (8) for $x$, it is enough to show that

$$
\operatorname{pr}_{\mathbf{E}}^{*} H^{0}\left(\mathbf{E} / \mathbf{H}, \Omega_{\mathbf{E} / \mathbf{H}}\right) \subset\left(p / E_{p-1}(\underline{\mathbf{E}}, \omega)\right) H^{0}\left(\mathbf{E}, \Omega_{\mathbf{E}}\right)
$$

where $\omega$ denotes any non-vanishing one-form on $\mathbf{E}$. By Theorem 3.1 in $\mathrm{Ka}$, the natural projection $\operatorname{pr}_{\mathbf{E}}: \mathbf{E} \rightarrow \mathbf{E} / \mathbf{H}$ reduces, modulo $p / E_{p-1}(\underline{\mathbf{E}}, \omega)$, to $\operatorname{Fr}_{p}$. Since pulling back via $\operatorname{Fr}_{p}$ kills one-forms the claim follows in this case.

Next, we deal with $x \in \mathcal{V}[1,1]$, i.e., with the case $\left|E_{p-1}(x)\right|=1$. Indeed we have already proven the statement for all such $x$ of good reduction. Recall the map $\pi: \mathcal{V}[1,1] \rightarrow \mathcal{Z}$ defined in the paragraph before this lemma. Let $y=\pi(x) \in \mathcal{Z}$. Denote by $\tilde{y}: \operatorname{Spf}\left(\mathcal{O}_{L}\right) \rightarrow \tilde{Z}$ the formal lifting of $y$ to the formal model $\tilde{Z}$ of $\mathcal{Z}$. Then the formal lifting of $\pi(\tau x)=\sigma y$ (see Equation (6) ) is $\tilde{\sigma} \tilde{y}$. Since $\underline{\omega}=\pi^{*} \underline{\omega}, \underline{\omega}^{\prime}=\pi^{*} \underline{\omega}^{\prime}$, and by Equation (7) (and by integral versions of such compatibilities) it is straightforward to see that (8) for $x$ is equivalent to

$$
\operatorname{pr}_{\tilde{y}}^{*}\left(H^{0}\left(\operatorname{Spf}\left(\mathcal{O}_{L}\right),(\tilde{\sigma} \tilde{y})^{*} \underline{\omega}=\tilde{y}^{*} \underline{\omega}^{\prime}\right)\right) \subset p\left(H^{0}\left(\operatorname{Spf}\left(\mathcal{O}_{L}\right), \tilde{y}^{*} \underline{\omega}\right)\right)
$$


where $\underline{\omega}$ and $\underline{\omega}^{\prime}$ are on $\tilde{Z}$, and $\operatorname{pr}_{\tilde{y}}^{*}$ is the specialization of $\operatorname{pr}^{*}: \underline{\omega}^{\prime} \rightarrow \underline{\omega}$ via $\tilde{y}$. Hence, it suffices to show that pr* $\underline{\omega}^{\prime} \rightarrow \underline{\omega}$ on $\tilde{Z}$ reduces to the 0 morphism modulo $p$. Indeed, since this map is defined over $\mathbb{Z}_{p}$, it is enough to prove this statement over $\mathbb{Z}_{p}$. But on the non-cuspidal part of $\tilde{Z}_{\mathbb{Z}_{p}} \otimes \mathbb{F}_{p}$ (which is the ordinary part of $X_{1}(N) \otimes \mathbb{F}_{p}$ ) this map reduces, modulo $p$, to $\mathrm{Fr}_{p}^{*}:\left(\underline{\omega} \otimes \mathbb{F}_{p}\right)^{(p)} \rightarrow\left(\underline{\omega} \otimes \mathbb{F}_{p}\right)$ which is zero. Now, a morphism of invertible sheaves on an integral curve which is zero away from a finite number of points has to be zero. We are done.

Remark 3.4. One can prove that the inequality in Lemma 3.3 is indeed an equality, though we don't need it for our arguments.

Corollary 3.5. Let $h \in H^{0}\left(\mathcal{V}[1,1], \underline{\omega}^{k}\right)$. For all $n \geq 1$ we have

$$
\left|h^{\tau^{n}}\right|_{\mathcal{V}[1,1]} \leq|h|_{\mathcal{V}[1,1]}<\infty
$$

Proof. This follows from Lemma 3.3 with $r=1$, and Lemma 2.2 .

\section{Classicality of overconvergent modular forms}

Recall that we are in level $\Gamma_{1}\left(N p^{m}\right)$ with $N>4,(p, N)=1$, and $m>0$, and we denote $X_{1}\left(N p^{m}\right)_{K}^{\text {an }}, \mathcal{V}_{1}\left(N p^{m}\right)$, and $\mathcal{U}_{1}\left(N p^{m}\right)$ by $X_{K}^{\text {an }}, \mathcal{V}$, and $\mathcal{U}$ respectively. We prove the following theorem.

Theorem 4.1. Let $f$ be an overconvergent modular form of weight $k$ and level $\Gamma_{1}\left(N p^{m}\right)$ defined over $K$. Let $R(x)$ in $K[x]$ be a polynomial all roots of which in $\mathbb{C}_{p}$ have valuation less than $k-1$. If $R(\mathrm{U}) f$ is classical on $X_{1}\left(N p^{m}\right)_{K}$, then so is $f$.

Coleman's theorem follows as a result.

Corollary 4.2. (Coleman) Let $f$ be a generalized U-eigenform of weight $k$, level $\Gamma_{1}\left(N p^{m}\right)$, and slope less than $k-1$. Then $f$ is classical.

Proof. If $f$ is a generalized eigenform of weight $k$ and slope less than $k-1$, then for some polynomial $R(x)$ as in Theorem $4.1 R(\mathrm{U}) f$ equals 0 which is very well classical! Hence $f$ is classical.

4.1. Proof of the Theorem. We will now prove Theorem 4.1. First we prove the statement for $R(x)$ of degree 1 . Let $f$ be an overconvergent modular form of weight $k$ on $X_{K}^{\text {an }}$ such that for some $a \in K$ of valuation less than $k-1$, we can extend Uf - af to a section $F$ of $\underline{\omega}^{k}$ on $X_{K}^{\text {an }}$. By Buzzard's theorem 3.1 one can extend $f$ to $\mathcal{U}$. Since $\{\mathcal{U}, \mathcal{V}\}$ is an admissible covering of $X_{K}^{\text {an }}$, to prove the classicality of $f$, it is enough to show that the restriction of $f$ to $\mathcal{V} \cap \mathcal{U}=\mathcal{V}[t, 1)$ (which we still denote by $f$ ) can be extended to $\mathcal{V}=\mathcal{V}[t, 1]$. Let $b=p^{k-1} / a$. By definition of $\tau$, we know that $f-b f^{\tau}$ is a section of $\underline{\omega}^{k}$ on $\mathcal{V}\left[t^{1 / p}, 1\right)$.

Proposition 4.3. The section $f-b f^{\tau} \in H^{0}\left(\mathcal{V}\left[t^{1 / p}, 1\right), \underline{\omega}^{k}\right)$ extends to a section $F_{1}$ of $\underline{\omega}^{k}$ on $\mathcal{V}\left[t^{1 / p}, 1\right]$. 
Proof. First we define $F_{1}$ away from the cusps. Let $(E, i, P) \in \mathcal{V}\left[t^{1 / p}, 1\right]-\{$ cusps $\}$. Let $C$ be any subgroup of $E$ of order $p$ which is different from $H_{1}$ and which intersects $\langle P\rangle$ trivially, i.e., $C \neq\left\langle p^{m-1} P\right\rangle$. Then the image of $p^{m-1} P$ in $E / C$ generates the canonical subgroup of $E / C$. The reason is that in general if $E$ has a canonical subgroup, then for any non-canonical subgroup $C$ of $E, E / C$ has a canonical subgroup which equals $E[p] / C$. See Theorem 3.10.7.3 in [Ka]. Therefore $(E / C, \bar{i}, \bar{P}) \in \mathcal{U}$, and hence $f(E / C, \bar{i}, \bar{P})$ is defined. Define

$$
F_{1}(E, i, P):=(1 / a p) \sum_{C}\left(\operatorname{pr}^{*}\right)^{k} f(E / C, \bar{i}, \bar{P})-(1 / a) F(E, i, P)
$$

where $C$ runs through the subgroups of $E$ of order $p$, which are different from $H_{1}$, and intersect $\langle P\rangle$ trivially. Then on $\mathcal{V}\left[t^{1 / p}, 1\right]-\{$ cusps $\}$ we have

$$
\begin{aligned}
F_{1}(E, i, P) & =(1 / a p) \sum\left(\mathrm{pr}^{*}\right)^{k} f(E / D, \bar{i}, \bar{P})-(1 / a p)\left(\mathrm{pr}^{*}\right)^{k} f\left(E / H_{1}, \bar{i}, \bar{P}\right)-(1 / a) F(E, i, P) \\
& =(1 / a) \mathrm{U} f(E, i, P)-b f^{\tau}(E, i, P)-(1 / a) F(E, i, P) \\
& =f(E, i, P)-b f^{\tau}(E, i, P)
\end{aligned}
$$

where in the above $D$ runs through all subgroups of $E$ of order $p$ which intersect $\langle P\rangle$ trivially. This proves that $F_{1}$ extends $f-b f^{\tau}$ on $\mathcal{V}\left[t^{1 / p}, 1\right]-\{c u s p s\}$. To end the proof, we show that $F_{1}$ extends to $\mathcal{V}\left[t^{1 / p}, 1\right]$. We calculate the $q$-expansion of $F_{1}$ around the cusps in $\mathcal{V}$. For this, we can assume that $K$ contains a primitive $p^{m}$-th root of unity $\zeta_{p^{m}}$. The cusps in $\mathcal{V}$ consist of all isomorphism classes of $\left(\operatorname{Tate}(q), i, q^{A / p^{m}} \zeta_{p^{m}}^{B}\right)$, where $i: \mu_{N} \rightarrow \operatorname{Tate}(q)$ is an inclusion, and $A$ is not divisible by $p$. We show the analyticity around the cusp $c$ corresponding to $A=1, B=0$. The other cases are similar. Let $\zeta_{p}=\left(\zeta_{p^{m}}\right)^{p^{m-1}}$.

$$
\begin{aligned}
F_{1}(c) & =(1 / a p) \sum_{j=1}^{p-1}\left(\operatorname{pr}^{*}\right)^{k} f\left(\operatorname{Tate}(q) /\left\langle q^{1 / p} \zeta_{p}^{j}\right\rangle, \bar{i}, \bar{q}^{1 / p^{m}}\right)-(1 / a) F(c) \\
& =(1 / a p) \sum_{j=1}^{p-1} f\left(\operatorname{Tate}\left(q^{1 / p} \zeta_{p}^{j}\right), i, q^{1 / p^{m}}\right)-(1 / a) F(c)
\end{aligned}
$$

But (Tate $\left.\left(q^{1 / p} \zeta_{p}^{j}\right), i, q^{1 / p^{m}}\right)$ can be obtained from $c_{j}^{\prime}=\left(\operatorname{Tate}(q), i, q^{1 / p^{m-1}} \zeta_{p^{m}}^{-j}\right)$ via a base extension sending $q$ to $q^{1 / p} \zeta_{p}^{j}$. For $j$ not divisible by $p$, we have $c_{j}^{\prime} \in \mathcal{U}$. The reason is that we have $\left(q^{1 / p^{m-1}} \zeta_{p^{m}}^{-j}\right)^{p^{m-1}}=\zeta_{p}^{-j}$ which generates the canonical subgroup of $\operatorname{Tate}(q)$. Therefore, $f$ is analytic at $c_{j}^{\prime}$. Furthermore $F$ is also analytic at $c$. Hence $f$ can be extended to the cusp $c$.

We now set the stage for the application of our gluing lemma. For each $n \geq 1$ we define a section $F_{n} \in H^{0}\left(\mathcal{V}\left[t^{1 / p^{n}}, 1\right], \underline{\omega}^{k}\right)$ by

$$
F_{n}=\sum_{i=0}^{n-1} b^{i} F_{1}^{\tau^{i}} .
$$

By Proposition 4.3 we have $F_{\left.1\right|_{\mathcal{V}\left[t^{1 / p}, 1\right)}}=f-b f^{\tau}$, and it follows that on $\mathcal{V}\left[t^{1 / p^{n}}, 1\right)$ we have

$$
F_{\left.n\right|_{\mathcal{V}\left[t^{1 / p^{n}}, 1\right)}}=f-b^{n} f^{\tau^{n}}
$$


Since $v(b)>0$, Corollary 3.5 and Lemma 2.2 imply that

$$
g:=\sum_{i \geq 0} b^{i} F_{1}^{\tau^{i}}
$$

converges to a section of $\underline{\omega}^{k}$ on $\mathcal{V}[1,1]$. This is exactly the infinite sum that was met in the Introduction (for the case $m=1$ ). For each $n \geq 1$ we have

$$
F_{\left.n\right|_{\mathcal{V}[1,1]}}=g-b^{n} g^{\tau^{n}} .
$$

We now want to apply Lemma 2.3 to show that $f$ and $g$ glue together to form a section $\mathbf{f}$ of $\underline{\omega}^{k}$ on $\mathcal{V}$. Then $\mathbf{f}$ (defined on $\mathcal{V}$ ) will agree over $\mathcal{V} \cap \mathcal{U}$ with $f$ (defined on $\mathcal{U}$ ), and we will get the desired extension of $f$ to the whole modular curve.

We will apply the gluing lemma with $X=X_{1}\left(N p^{m}\right)_{\mathcal{O}_{K}}, M=\underline{\omega}^{k}, \mathcal{X}=\mathcal{V}$ which is a smooth affinoid subdomain of $\tilde{X}_{\text {rig }}=X_{K}^{\text {an }}, \mathcal{Y}=\mathcal{V}[t, 1), \mathcal{Z}=\mathcal{V}[1,1], \overline{\mathcal{Z}}_{n}=\mathcal{V}\left[t^{1 / p^{n}}, 1\right]$. Since we have $F_{n}-f=-b^{n} f^{\tau^{n}}, F_{n}-g=-b^{n} g^{\tau^{n}}$, and since $v(b)=k-1-v(a)>0$, to verify the conditions of the Lemma, it's enough to show that $\left\{\left|g^{\tau^{n}}\right|_{\mathcal{V}[1,1]}\right\}_{n}$ and $\left\{\left|f^{\tau^{n}}\right|_{\mathcal{V}\left[t^{1 / p^{n}}, 1\right)}\right\}_{n}$ are uniformly bounded. The first assertion follows from Corollary [3.5. In the following we prove the second assertion.

Lemma 4.4. The section $f$ is bounded on $\mathcal{V}[t, 1)$.

Proof. Since $f-b f^{\tau}$ extends to $\mathcal{V}\left[t^{1 / p}, 1\right]$ which is an affinoid, $\left|f-b f^{\tau}\right|_{\mathcal{V}\left[t^{1 / p}, 1\right)}$ is finite by Lemma 2.2. Similarly, $|f|_{\mathcal{V}\left[t, t^{1 / p}\right]}$ is finite since $\mathcal{V}\left[t, t^{1 / p}\right]$ is an affinoid. Let $M_{1}$ be a common upper bound. We show, by induction on $n$, that $f$ is bounded by $M_{1} t^{-k\left(1 / p+\ldots+1 / p^{n}\right)}$ on $\mathcal{V}_{n}:=\mathcal{V}\left[t^{1 / p^{n}}, t^{1 / p^{n+1}}\right]$ for all $n$. Let $x \in \mathcal{V}_{n+1}$. Then $\tau(x) \in \mathcal{V}_{n}$ and by the induction hypothesis and Lemma 3.3 we have

$$
\begin{aligned}
\left|f^{\tau}(x)\right| & \leq|f(\tau(x))|\left|E_{p-1}(x)\right|^{-k} \\
& \leq M_{1} t^{-k\left(p^{-1}+\ldots+p^{-n}\right)} t^{-k p^{-(n+1)}}=M_{1} t^{-k\left(p^{-1}+\ldots+p^{-n}+p^{-(n+1)}\right)}
\end{aligned}
$$

So we can write

$$
\begin{aligned}
|f|_{\mathcal{V}_{n+1}} & \leq \max \left\{\left|f-b f^{\tau}\right|_{\mathcal{V}_{n+1}},\left|b f^{\tau}\right|_{\mathcal{\nu}_{n+1}}\right\} \\
& \leq \max \left\{M_{1},\left|f^{\tau}\right|_{\mathcal{V}_{n+1}}\right\} \\
& \leq M_{1} t^{-k\left(p^{-1}+\ldots+p^{-n}+p^{-(n+1)}\right)} .
\end{aligned}
$$

Now it is clear that $f$ on $\mathcal{V}[t, 1)=\bigcup_{n \geq 1} \mathcal{V}_{n}$ is bounded by

$$
\sup _{n}\left\{M_{1} t^{-k\left(p^{-1}+\ldots+p^{-n}+p^{-(n+1)}\right)}\right\}=M_{1} t^{-k /(p-1)}=: M_{2} .
$$

We are now able to prove the desired uniform boundedness.

Lemma 4.5. There is an $M>0$ such that for all $n \geq 0$ we have

$$
\left|f^{\tau^{n}}\right|_{\mathcal{V}\left[t^{\left.1 / p^{n}, 1\right)}\right.} \leq M
$$

Proof. Let $x \in \mathcal{V}\left[t^{1 / p^{n}}, 1\right)$. By Lemma 3.3, we have

$$
\left|f^{\tau^{n}}(x)\right| \leq\left|f^{\tau^{n-1}}(\tau(x))\right|\left|E_{p-1}(x)\right|^{-k} .
$$


Inductively, we find

$$
\left|f^{\tau^{n}}(x)\right| \leq\left|f\left(\tau^{n}(x)\right)\right|\left(\prod_{j=0}^{n-1}\left|E_{p-1}\left(\tau^{j}(x)\right)\right|\right)^{-k} .
$$

Now $x \in \mathcal{V}\left[t^{1 / p^{n}}, 1\right)$ satisfies $\left|E_{p-1}(x)\right| \geq t^{1 / p^{n}}$. Theorem 3.10.7(2) in $[\mathrm{Ka}$ ] that we have implies $\left|E_{p-1}\left(\tau^{j}(x)\right)\right|=\left|E_{p-1}(x)\right|^{p^{j}}$. Also, since $\tau^{n}(x) \in \mathcal{V}[t, 1)$, by Lemma 4.4 we find that $\left|f\left(\tau^{n}(x)\right)\right| \leq M_{2}$. Putting all this together, we have

$$
\left|f^{\tau^{n}}(x)\right| \leq M_{2}\left|E_{p-1}(x)\right|^{-k\left(p^{n}-1\right) /(p-1)} \leq M_{2} t^{-k\left(p^{n}-1\right) / p^{n}(p-1)} \leq M_{2} t^{-k /(p-1)}=: M .
$$

This proves Theorem 4.1 for $R(x)$ of degree one. Here is how we deal with the general case: It's enough to prove the theorem over a finite extension of $K$, and hence, we can assume $R(x)=\left(x-a_{1}\right)\left(x-a_{2}\right) \ldots\left(x-a_{l}\right)$, such that $v\left(a_{j}\right)<k-1$ for all $j$. We have

$$
\left(\mathrm{U}-a_{1}\right)\left(\mathrm{U}-a_{2}\right) \ldots\left(\mathrm{U}-a_{l}\right) f=F .
$$

Let $f_{j}=\left(\mathrm{U}-a_{j+1}\right)\left(\mathrm{U}-a_{j+2}\right) \ldots\left(\mathrm{U}-a_{l}\right) f$. Then for $f_{1}$ we have $\left(\mathrm{U}-a_{1}\right) f_{1}=F$ and hence by the above $f_{1}$ is classical. For $f_{2}$ we have $\left(\mathrm{U}-a_{2}\right) f_{2}=f_{1}$ and since $f_{1}$ is classical, we deduce that $f_{2}$ is classical. Continuing this way we get that $f$ is classical. The proof of Theorem 4.1 is now complete.

\section{REFERENCES}

[Ba] W. Bartenwerfer, Die erste "metrische" Kohomologiegruppe glatter affinoider Räume. (German) Nederl. Akad. Wetensch. Proc. Ser. A 40 (1978), no. 1, 1-14.

[BL] S. Bosch; W. Lütkebohmert, Formal and rigid geometry. I. Rigid spaces. Math. Ann. 295 (1993), no. 2, 291-317.

[Bu] K. Buzzard, Analytic continuation of overconvergent eigenforms. J. Amer. Math. Soc. 16 (2003), no. 1, 29-55.

[BT] K. Buzzard; R.Taylor, Companion forms and weight one forms. Annals of Math. (2) 149 (1999), no. 3, 905-919.

[Co1] R. F. Coleman, Classical and overconvergent modular forms. Inventiones Math. 124(1996), 215-241.

[Co2] R. F. Coleman, Classical and overconvergent modular forms of higher level. J. Théor. Nombres Bordeaux 9(1997), no. 2, 395-403.

[HT] M. Harris; R. Taylor, The geometry and cohomology of some simple shimura varieties. Annals of Mathematics Studies, Princeton University Press, Princeton, N.J., 2001.

[Kas1] P. L Kassaei, p-adic modular forms over Shimura curves over $\mathbb{Q}$. Thesis. M.I.T., 1999.

[Kas2] P. L Kassaei, P-adic modular forms over Shimura curves over totally real fields. Compositio Math. 140 (2004), no. 2, 359-395.

[Ka] N. Katz, p-adic properties of modular schemes and modular forms, Modular forms in one variable III. Lecture Notes in Mathematics, vol. 350, Springer Verlag, (1973), 69-190.

[KM] N. Katz; B. Mazur, Arithmetic moduli of elliptic curves. Annals of Mathematics Studies, 108. Princeton University Press, Princeton, N.J., 1985.

[KL] M. Kisin; K.F. Lai, Overconvergent Hilbert Modular Forms. To appear in Amer. J. Math., 39 pp. 2004.

Department of Mathematics, McGill University, Montreal, QC, H3A 2K6, Canada

E-mail address: kassaei@math.mcgill.ca 